\title{
La nueva agenda de derechos humanos para la igualdad y el desarrollo humano sostenible.
}

\section{Luis Carlos Herrera $\mathbf{M}^{1, *}$}

${ }^{1}$ Investigador, Facultad de Ciencias Sociales, Universidad Católica Santa María la Antigua, Panamá, República de Panamá.

*Autor para correspondencia. Email: lherreram@usma.com.pa

Recibido: 27 de noviembre de 2015

Aceptado: 4 de diciembre de 2015

\begin{abstract}
The interest of first generation of human rights was to incorporate fundamental freedoms, civil, economic and political rights of the individual, to limit the power of the state. The second generation rights are of social content to ensure the best living conditions for people. Third generation rights are rights or collective rights of peoples. A new human rights agenda, is the only way to achieve sustainable development, where the human being is the center of the action nationally, regionally and globally; must be holistic, articulating, universal, indivisible, multicultural, multi-ethnic, gender-sensitive, inclusive, with citizen participation, legal security, solidarity and international cooperation; including strategies and public policies to respect and guarantee the rights of all people, with special protection to vulnerable groups. The elimination of the violation of human rights; gender discrimination, violence against women, insecurity, hunger, poverty, environmental degradation, social inequality, lack of access to justice, migration, the effects of climate change, food security, education excellence, holistic health, water, housing, are priorities in a new agenda.
\end{abstract}

Keywords: Human Rights; Human Development; Sustainable Development; Gender; Inequality.

\section{Resumen}

En los derechos humanos de primera generación; el interés era incorporar las libertades fundamentales, los derechos civiles, patrimoniales y políticos del individuo, para poner límites al poder del Estado. Los derechos de segunda generación son de contenido social para procurar las mejores condiciones de vida a las personas. Los derechos de tercera generación son derechos colectivos o derechos de los pueblos. Una nueva agenda de derechos humanos es la única forma de alcanzar un desarrollo sostenible, donde el ser humano sea el centro del actuar nacional, regional y global. Debe ser holística, articuladora, universal, indivisible, multicultural, pluriétnica, con enfoque de género, inclusiva, con participación ciudadana, seguridad jurídica, solidaridad y cooperación internacional; que incluya estrategias y políticas públicas de respeto y garantía de los derechos de todas las personas, con especial 
protección a los grupos vulnerables. La eliminación de la violación a los derechos humanos — discriminación de género, violencia contra la mujer, inseguridad, hambre, pobreza, el deterioro al medio ambiente, desigualdad social, la falta de acceso a la justicia, la migraciones, los efectos del cambio climático, la seguridad alimentaria, la educación de excelencia, la salud integral, el agua, la viviendason prioridades en una nueva agenda.

Palabras clave: Derechos Humanos; Desarrollo Humano; Género; Desigualdad; Desarrollo Sostenible.

\section{Introducción}

En América Latina y el Caribe encontramos una diversidad étnica, territorial y cultural, profundas desigualdades, debilidad institucional, transición demográfica; a la vez, contamos con un patrimonio natural, cuya protección garantiza un equilibrio entre el ambiente y las personas. No obstante, es preocupante que, a pesar de las obligaciones de los Estados de garantizarlos, es constante la grave violación a los derechos humanos, civiles, políticos, económicos, sociales, culturales, ambientales y de los pueblos.

Esta realidad hace obligante impulsar una agenda de derechos humanos que, por un lado, fortalezca el rol de los Estados para que tengan las capacidades y destinen los recursos necesarios para garantizar los derechos humanos a la población y, por el otro, que sea integral, solidaria, sostenible y sobre todo con rostro humano.

Las asimetrías entre países y particularidades nacionales y regionales; no puede esgrimirse como argumento válido para continuar postergando la obligación de cumplir la agenda de derechos humanos. Si bien tenemos que reiterar temas pendientes, es imperativo poner los acentos en temas relevantes, $y$ exigir a los Estados saldar la mora en sus obligaciones de garantizar a la población las distintas generaciones de derechos humanos, que son vitales para la vida, la integridad, la dignidad del ser humano y la eliminación de la desigualdad.

Lo que se propone es una agenda de derechos humanos para la igualdad y el desarrollo humano sostenible, más inclusiva, centrada en las personas para garantizar un buen vivir-con seguridad, integridad, empoderamiento, participación ciudadana, respetando la multiculturalidad, los derechos colectivos, la seguridad alimentaria y ambiental —en un Estado que los garantice y reconozca que sin el enfoque de respeto, protección y promoción de los derechos humanos estaremos condenados a repetir décadas de errores de políticas públicas, que no resuelven los problemas de la sociedad.

Es un reto alcanzar una agenda que genere modelos económicos, sociales y políticos transformadores, donde los países desarrollados deben dar muestra de su vocación de paz y solidaridad internacional, y los consensos no sean determinados por los intereses de seguir acumulando riqueza a costa de la miseria, el incremento de la indigencia y la desigualdad. 
Invest. pens. crit.

Vol. 3, No. 3, septiembre-diciembre 2015

pp. 38-61

Estamos convencidos que una de las contribuciones necesarias en la agenda del desarrollo post 2015, es la visión desde los derechos humanos, para que las decisiones y estrategias a futuro, estén contempladas en las políticas públicas, que nos permitan fortalecer a los Estados como garante de los derechos de las personas, que logren sociedades incluyentes, igualitarias, donde existan empleos decente, salud integral, educación de calidad, con equidad universal y gratuita; ambientes saludables, ciudades seguras, participación ciudadana, transparencia y rendición de cuentas.

Los derechos humanos, sociales, políticos, culturales, ambientales y tecnológicos de los pueblos, deben trascender los aspectos formales o legales. Es necesario que éstos estén inmersos en todas las estrategias que se acuerden para garantizar el desarrollo humano sostenible; que permitan consolidar una práctica cotidiana en las sociedades, con Estados que garanticen los derechos de las persona a los beneficios el desarrollo.

Podríamos hacer una lista interminable de los temas que nos preocupan, acordar objetivos, metas y acciones; pero si no existe un compromiso serio para que sean parte de políticas públicas que atiendan integralmente problemas prácticos y estratégicos de la población vulnerable y se destinen recursos para su ejecución, pasaría a ser una burla.

Según la Propuesta de agenda regional, preparada por la CEPAL en colaboración con el UNFPA, es necesario poner el acento en siete áreas temáticas para poder enfrentar los desafíos en materia de población de cara al futuro y reforzar su integración en las políticas y los programas de desarrollo económico y social. Estas áreas son: los derechos y necesidades de los niños, adolescentes y jóvenes; envejecimiento, protección social y desafíos socioeconómicos; igualdad de género; brechas en el acceso universal a los servicios de salud sexual y reproductiva; la migración internacional y la protección de los derechos de todas las personas migrantes; desigualdad territorial, movilidad espacial y vulnerabilidad ambiental; y derechos de pueblos indígenas y afrodescendientes. (Dirk Jasper-Faijer, 2014).

La nueva agenda de los derechos humanos es un compromiso de corto, mediano y largo plazo, que debe generar un pacto político, económico y social para cerrar las brechas de la desigualdad, potenciar y empoderar a las personas, bajo un enfoque holístico. En ese sentido esta agenda la planteamos en cinco (5) dimensiones:

1. Los derechos humanos, civiles y políticos; económicos, sociales y culturales; ambientales y colectivos.

2. Igualdad entre los géneros y eliminación de todas las formas de discriminación.

3. Derechos de los grupos más vulnerables, poblaciones indígenas y afrodescendientes.

4. Protección y prevención de los efectos del cambio climático y la seguridad alimentaria.

5. Participación ciudadana y transparencia. 


\section{Los derechos humanos, civiles y políticos; económicos, sociales y culturales, ambientales y colectivos.}

Los derechos humanos son inherentes a todos, son interrelacionados, interdependientes, indivisibles, universales e inalienables. Estos derechos forman parte del derecho internacional y están consagrados en las constituciones políticas de los países, como derechos individuales y sociales y gozan de regulaciones en el derecho interno. Son fuentes de deberes y derechos para la población, pero cuya responsabilidad principal recae en el Estado.

Han transcurridos varias décadas desde que los Estados asumen la responsabilidad de garantizar los derechos humanos. Se han creado protocolos y mecanismos de seguimiento de las convenciones y pactos para velar por el cumplimiento de estos derechos; y se incrementa acciones de la sociedad civil, organizaciones y activistas de derechos humanos, en su promoción y defensa.

Es hora de alcanzar una agenda de derechos humanos transformadora, capaz de guiar cada uno de los objetivos que se propongan en adelante, revisando concepciones y adoptando acuerdos; partiendo de la voluntad consciente de cambiar la realidad y los escenarios de desigualdad y discriminación que son una amenaza a la vida, la paz social, la democracia y devolver la confianza de los ciudadanos en sus instituciones. Es un reto para los tomadores de decisiones definir acciones y estrategias que permitan resolver los problemas a la población para un desarrollo humano sostenible con respeto a sus derechos humanos.

Según el documento la Nueva Era Demográfica de América Latina y el Caribe, elaborado por el Centro Caribeño de Población y Desarrollo (CELADE), división de la CEPAL, la población se triplicó en las pasadas seis décadas de 167 millones de personas de 1950 a 590 millones en el 2010; en el mismo tiempo la población del planeta creció de 2,500 a 7,000 Millones. Actualmente la población de la región llega a los 600 millones de personas y para 2050 llegaría a 751 millones de personas. (Miller, Saad, Martínez y Calvo, 2014).

Al revisar los temas de los derechos humanos civiles y políticos; económicos, sociales y culturales; ambientales y colectivos en América Latina, debe tener presente que existe una transformación en la población, particularmente en la estructura de la edad. A diferencia de Europa, por ejemplo, tenemos una población joven, en edad productiva, lo que se identifica como bono demográfico que debe ser aprovechada con políticas que les permita tener acceso a una educación de calidad, empleo no precarizado. Una agenda que no dé la espalda a los temas de la población vulnerable, especialmente la niñez, adolescencia, la juventud y la mujer.

Una de las preocupaciones para las sociedades en América Latina es lo relacionado a la juventud, que según el Fondo de Población del Sistema de Naciones Unidas, 
Invest. pens. crit.

Vol. 3, No. 3, septiembre-diciembre 2015

pp. 38-61

"Requieren de capacidades, espacios y protección para ser conductores de un proceso de desarrollo económico y social que permita, a la vez, reducir la pobreza y los abismantes índices de desigualdad socioeconómica, que atentan contra la estabilidad y la convivencia.

La pobreza que afecta a casi un tercio de la población joven de la región. Un 39\% de las y los jóvenes de 15 a 29 años se encuentra en la pobreza y casi un $10 \%$ en la indigencia. La pobreza juvenil en el área rural es de más del 46\%, duplicando la de la zona urbana que es del 25\%. La tasa de adolecentes embarazada de la región sólo es superada por África. El 20\% de los partos en la región correspondió a mujeres menores de 20 años de edad. El porcentaje de nacidos vivos del total de madres adolescentes de entre 15 y 19 años en algunos países de América Latina promedia el 18\%. En la región son madres el $9.1 \%$ de adolescentes urbanas y el 13\% de adolescentes rurales." (UNFPA, 2008, "Nuestro desafío", para.1-2).

Uno de los desafíos sigue siendo eliminar los niveles de pobreza e indigencia. La CEPAL señala que, para el 2011, el 31\% de la población en nuestra región se encontraba bajo la línea de pobreza y se reconoce como uno de los índices más bajo desde hace varias décadas. El abismo que se cierne sobre millones de latinoamericanos en condiciones de desigualdad social, de género, etnia, y territorio, nos colocan en el deshonroso primer lugar del mundo; "tomando el promedio simple regional, el ingreso medio captado por el 20\% más rico de la población, era más de 17 veces el del quintil más pobre y en los países con mayor desigualdad, el ingreso del quintil más rico, podría exceder en más de 25 veces el del quintil más pobre". (Dirk Jasper-Faijer, 2014).

Tenemos que exigir acciones contundentes, políticas publicas centradas en el ser humano, una ética en la gestión pública, anteponer a las personas a los intereses económicos y políticos del sistema, para trazar un camino de esperanza y de derechos, ya no hay tiempo para seguir debatiendo, es tiempo de asumir responsabilidades. La economía crece para unos pocos y la pobreza aumenta para muchos, eliminar la desigualdad y erradicar la pobreza, sigue siendo una de las metas importantes por alcanzar.

Con el propósito de generar una reflexión sobre los énfasis en la nueva agenda, en este primer punto correspondiente a los derechos humanos, civiles y políticos, sociales, económicos, culturales, ambientales, y de la colectividad; nos referimos solo a los temas que aún constituyen desafíos y aquellos que tienen serias implicaciones sobre la vida de las personas y el futuro de la convivencia humana. Como lo son:

1.1. Educación de calidad inclusiva y con equidad.

1.2. Salud integral y acceso a los servicios.

1.3. Violencia contra las mujeres, niñas y adolescentes.

1.4. Migración internacional.

Lo que no significa de modo alguno que los demás no sean esenciales. 


\subsection{Educación de calidad, inclusiva y con equidad.}

El derecho humano a una educación pública, gratuita y universal, orientada a la justicia social y ambiental, con calidad, inclusiva y de respeto a la dignidad de todas las personas, es un tema de primer orden en la agenda de derechos humanos. Desde la Declaración Universal de los Derechos Humanos en 1948, se reconoce el derecho a la educación, que se reitera en la Convención de los derechos del Niño en 1989, en Jomtien en Tailandia en 1990, se reconoce este derecho humano fundamental, sin distingos de edad para poder participar activamente en la sociedad, en Dakar, Senegal, en el año 2000, se reafirma ese compromiso con la educación para todos, compromiso que se incluye en los Objetivos de la Declaración del Milenio, en el 2000, para lograr la enseñanza primaria universal y la igualdad de género en la educación.

Pese a los distintos compromisos ratificados por los Estados, a lo largo de muchas décadas, entre avances y retrocesos en materia educativa, es un uno de los derechos que sigue siendo un desafio en muchos países. Los niveles educativos en nuestra región pueden ser evaluados de distintas formas, por calidad, desarrollo, accesibilidad, entre otros. Un tercio de los y las jóvenes entre 15 y 29 años de América Latina y El Caribe no asisten a ningún centro educativo. Los indígenas y afrodescendientes jóvenes de zonas rurales muestran menor finalización de estudios en comparación con otros grupos. (UNFPA, 2008)

En el año 2000, 164 gobiernos en Dakar acuerdan un marco de acción para alcanzar las metas de Educación para Todos (EPT), y así lograr una educación básica de calidad, sin exclusiones para todos los niños, niñas, jóvenes y adultos, que les permita actuar en los diferentes ámbitos de la vida social y ejercer la ciudadanía. La existencia de exclusión en la educación, con sesgos discriminatorios, impide a grandes segmentos de la población tener la posibilidad de terminar todos los niveles de la educación.

Según la Agenda Educacional Post-2015, de América Latina y el Caribe:

Se han observado altos niveles de desigualdad en todos los países, donde algunos de los factores determinantes son las clases social, el origen étnico, y la ubicación geográfica. Aun cuando aquellos en situación más precaria son capaces de subir en la escala social, cuando se compara su situación con los grupos más privilegiados, esta no ha mejorado significativamente. (UNESCO, 2015.Pág.5)

El derecho humano a la educación, debe replantearse en la nueva agenda como prioridad; no sólo se trata de alfabetizar, evitar la deserción escolar, los fracasos, modificar los modelos pedagógicos, aumentar la inversión pública y privada en educación, o resolver problemas de infraestructura escolar. Se requiere reflexionar y decidir, cuál es la excelencia académica que queremos alcanza, para ello hay que destinar esfuerzos para mejorar el perfil del docente; revisar el contenido curricular que incorpore el enfoque de derechos humanos, la igualdad con equidad de género, que los empodere, los forme con una consciencia critica, creativa y reflexiva, con valores y principios humanistas y uso de las tecnologías. 
Invest. pens. crit.

Vol. 3, No. 3, septiembre-diciembre 2015

pp. 38-61

Hay temas relacionados con otros derechos, que deben ser parte de las políticas públicas en materia educativa, que no pueden verse solamente como un problema de salud; nuestra niñez, adolescencia y juventud, requiere que su derecho sexuales y reproductivos se vean reflejados en una educación integral en la sexualidad, tomando en cuenta su desarrollo biopsicosocial, que les incluya temas como la afectividad, la igualdad de género, autocuidado y protección, rechazo ante todas las formas de violencia.

Entre los aspectos que los Estados deben garantizar con prioridad es la educación primaria gratuita y obligatoria para todos los niños y las niñas y la garantía de que el contenido educativo es acorde con los principios de los derechos humanos. Educar para la igualdad y la equidad de género, el respeto a la diversidad y la comprensión de la no discriminación y la multiculturalidad, permitirá formar un nuevo ser humano. La dimensión ética, la responsabilidad global, ambiental, el desarrollo de las capacidades en el uso de las tecnologías, debe trasnverzalizar los contenidos.

En este siglo la educación primaria no es suficiente, se debe procurar el mayor acceso a la educación en todos los niveles, (secundaria, técnica, profesional y/o superior). Un ejemplo de la indivisibilidad de los derechos humanos y su universalidad, es el derecho a la educación, este derecho esta de forma permanente en toda la vida de los seres humanos, y de él dependen el acceso a otros derechos y facilitan la movilidad social, eliminar el circulo generacional y la feminización de la pobreza, disminuye los riesgos de la violación de otros derechos humanos y la discriminación.

\subsection{Salud integral y Acceso a los servicios.}

El derecho a la salud integral con equidad, es uno de los temas que requiere intensificar esfuerzos. La salud, según la Organización Mundial de la Salud, es entendida como un estado de completo bienestar, físico, mental y social, y no solamente la ausencia de infecciones o enfermedades y se reconoce que es uno de los derechos fundamentales de los seres humanos, sin distinción de, raza, religión, ideas políticas, o condición económica y social.

La salud, como derecho-deber, implica que es responsabilidad del Estado y la sociedad: la ejecución de políticas de salud, no solo corresponde a las instituciones de salud, debe verse como políticas integrales. La educación y el trabajo deben articular acciones, porque es determinante para la salud si la población está más educada, y cuenta con fuentes de ingresos.

Pero esta aspiración a Salud integral para todos está lejos de ser cumplida en América Latina y el Caribe, donde el acceso efectivo a los servicios de salud, y la calidad de estos servicios, es una deuda pendiente con los sectores más vulnerables de la población, aunque se presentan diferencias en los indicadores de salud.

El Secretario General de la Organización de las Naciones Unidas, Ban Ki. Moon, en el informe de avances de los ODM, señala que se han logrado enormes avances en reducir la mortalidad infantil. Sin embargo indica que: 
En el 2012, la mayor parte de los 6,6 millones de muertes de menores de 5 años se debió a las principales enfermedades infeccionas, como neumonía, diarrea y paludismo...Muchas muertes de menores de 5 años, se produjeron en niños ya debilitados por nutrición insuficiente, que es un factor asociado a casi la mitad de las muertes en menores de 5 años a nivel mundial, principalmente en los países con bajos ingresos, donde la mal nutrición y las enfermedades infecciosas están altamente concentradas sobre todo en los sectores pobres. (Moon, 2014, Pág.25)

Las condiciones de pobreza y desigualdad presentes en la región, exigen atender el tema del derecho a la salud, de manera integral y con equidad, como prioridad del Estado.

En una región de tanta riqueza, la desigualdad la testimonian los más de 167 millones de personas viven en pobreza y 66 millones en la indigencia. La inequidad es claro indicador que esta población ve limitada su posibilidades de acceso a los servicios de salud. "La desigualdad está asociada a una menor esperanza de vidas, tasas de mortalidad infantil muy altas, menor altura, pobre salud autopercibida, peso más bajo al nacer, sida y depresión.” (Wikilson \& Picket, 2009, pág. 103).

Por ejemplo, podemos llevar los servicios de salud a una comunidad, donde se benefician todas las mujeres y hombres: allí estaríamos hablando de igualdad. Pero no es lo mismo el acceso a los servicios de salud de una mujer, o si además es indígena y adulta mayor. Allí entran los criterios de equidad en los servicios que requieren decisiones de acción afirmativa o positiva de derechos. De no establecerlos, esconderíamos otras desigualdades. "La equidad puede conducir a buscar una dimensión más exigente del igualdad”. (Fitoussi \& Rosanvallon, 2003)

Una de las demandas persistentes en nuestras comunidades es la falta de acceso a los servicios de salud con calidad y calidez, insuficiencia de personal de la salud (médicos de las diferentes especialidades, enfermeras, laboratoristas, educadores de la salud, etc.), camas hospitalarias, medicamentos, instalaciones y equipos; y muchas enfermedades y muertes, especialmente en la niñez y las mujeres serían prevenibles si se contara con estos servicios.

Otro de los problemas de la falta de acceso a los servicios de salud es la mortalidad materna, es decir, la muerte de la mujer, durante el embarazo, el parto, o dentro de los 42 días siguientes por cualquier causa relacionada, con el embarazo, el parto o el puerperio.

Según el Perfil de la Salud de las Mujeres y los Hombres en las Américas:

La mortalidad materna ha sido calificada como la prueba más clara de inequidad social. Así como la expresión más dramática de la desventaja que afecta a grandes sectores de mujeres de la región en el ejercicio de sus derechos fundamentales. Las muertes maternas son injustas, ya que esencialmente son prevenibles, Desde hace varias décadas se cuenta con el conocimiento y la tecnología necesarios para impedir estas defunciones, que se producen principalmente entre las mujeres pobres y marginadas. (Jara \& Roberts, 2009, Pág.55) 
Invest. pens. crit.

Vol. 3, No. 3, septiembre-diciembre 2015

pp. 38-61

Lo imperdonable de la situación de la salud en América y Latina y el Caribe, que muchas de las muertes maternas e infantiles eran prevenibles; y son causadas principalmente por la falta de acceso a la salud, calidad de la atención antes, durante y después del parto; las limitaciones a las infraestructuras, la desnutrición, falta de acceso a servicios de salud, el nulo o escaso personal de salud, especialmente en áreas de difícil acceso, la carencia de agua potable y saneamiento básico; y las condiciones de pobreza y extrema pobreza.

\subsection{Violencia contra las mujeres, niñas y adolescentes.}

La violencia contra las mujeres, niñas y adolescentes es un tema crucial de derechos humanos que amerita atenderse desde una dimensión ética, humana, y con determinación.

Uno de los tratados internacionales de derechos humanos en favor de la mujer, cuyo impacto ha sido relevante en el reconocimiento de los derechos de las mujeres, es la Convención para la Eliminación de todas las Formas de Discriminación contra la Mujer, (CEDAW, por sus siglas en inglés), que entró en vigencia en 1981, con carácter vinculante y cuyo Comité, creado desde 1992, ha recomendado a los Estados latinoamericanos y caribeños, aprobar planes de acción nacionales contra la violencia hacia la mujer, las niñas, adolescentes, jóvenes y adultas mayores y eliminar las causas estructurales de la discriminación, crear los mecanismos nacionales responsables de las políticas públicas, así como el revisar las normativas, incorporar medidas de acción afirmativas necesarias, eliminar los lenguajes sexistas, estereotipos y crear igualdad de oportunidades para que las mujeres logren el empoderamiento económico, político y físico.

Pese a este y otros esfuerzos de los Organismos del Sistema de Naciones Unidas, para prevenir y erradicar la violencia, y los llamados a los Estados por parte del Comité de Derechos Humanos, el Comité de los Derechos del Niño/a, Comité contra la Discriminación Racial, el de la Tortura, no se eliminan sus causas.

A través del Sistema Interamericano de la Convención Interamericana para Prevenir, Sancionar y erradicar todas las formas de Violencia contra las Mujeres (Belem Do Pará), aprobada en 1994, exige que los Estados adopten todas las medidas para su cumplimiento. Es la única Convención de esta naturaleza que cuenta con un Mecanismo de Seguimiento a la Convención (MESECVI) y un Comité de expertas de violencia, representantes de la sociedad civil o CEVI.

Han transcurrido veinte años de estar vigente esta Convención que rige a 34 Estados Latinoamericanos y Caribeños, la el cual es el camino que nos permitirá alcanzar la garantía plena de nuestros derechos humanos, y una vida libre de violencia para las mujeres. Podemos seguir debatiendo o valorando lo mucho o poco que nos falta para alcanzar algunas metas; pero el precio que se tiene que pagar es muy alto, si no se toman las decisiones correctas en esta nueva agenda, con una perspectiva, integral, inclusiva e intercultural, capaz de reconocer la diversidad y especificidades de las mujeres y niñas víctimas de la violencia. 
El estudio sobre planes y políticas para erradicar la violencia contra las Mujeres en América Latina y el Caribe del Programa de Naciones Unidas para el Desarrollo (PNUD) y la entidad de las Naciones Unidas para la Igualdad de Género y el Empoderamiento de las Mujeres (ONU Mujeres), en cuanto al Femicidio, señala lo siguiente:

Una mención especial merece el caso de femicidio, esta expresión fatal de la violencia ejercida hacia las mujeres aún resulta una asignatura pendiente den el 75\% de los países de la región, si consideramos que en solo 9 países de los 32 países estudiados se ha sancionado legislación que aborda penalmente el asesinato de las mujeres, por el hecho de ser mujeres. (Essayag, 2013, Pág.19)

La violencia, en cualquiera de sus manifestaciones, es un obstáculo para el desarrollo. El mundo se priva de contar con las contribuciones de la mitad de la población del planeta cuando no protege sus derechos humanos en todo su ciclo vital. La protección del derecho humano de vivir libre de violencia se agrava si se trata de mujeres pobres, indígenas, discapacitadas, migrantes, refugiadas, víctimas de trata, tráfico, trabajadoras sexuales, afrodescendientes, pertenecientes a otras minorías étnicas, entre otras.

La atención integral a la violencia contra la mujer requiere de un pacto social que reconozca a la violencia no solo como problema de salud pública, sino como grave violación de derechos humanos, de manera que se adopten políticas concertadas y planes nacionales, con amplia participación institucional, de las sociedad civil, empresas medios de comunicación social y organizaciones de mujeres.

Debe existir acceso a la justicia con equidad de género, acciones preventivas intersectoriales e integrales y para la atención a las víctimas y sus familias. También se necesita centros de orientación especializados y centros de acogida; destinar recursos económicos y humanos para desarrollar sus competencias y habilidades, generar más oportunidades para que alcancen su autonomía económica, así como crear observatorios o sistemas de vigilancia, implementar indicadores y recopilar datos desagregados sobre la violencia de género, incluyendo además de la violencia doméstica, femicidio y trata de mujeres y niñas.

Los derechos no pueden seguirse tratando aisladamente: el menoscabo de uno afecta a otros. La vinculación entre los derechos y los modelos económico y social requiere de situar a los seres humanos en la prioridad de las decisiones, para que se cumplan con todos y cada uno de sus derechos humanos. Seguir experimentando curas, sin entrar a resolver las causas de la enfermedad y sus complicaciones, es no alterar las condiciones existentes de violación a esos derechos.

\subsection{Migración internacional}

Nuestras poblaciones tienden a la migración en búsqueda de mejores oportunidades para sus familias. Los Estados deben generar normas recíprocas para que el proceso de travesía, inserción y retorno a sus países sea seguro. 
Invest. pens. crit.

Vol. 3, No. 3, septiembre-diciembre 2015

pp. 38-61

La promoción y protección de los derechos humanos debe amparar a las y los migrantes, los cuales deben someterse a muchos abusos que les afecta su vida, integridad y la de sus familias. Los problemas migratorios, lejos de reducirse, tienden a ser más complejos y aumentar la necesidad de adecuar la legislación, adoptar políticas, medidas y acuerdos nacionales y regionales, para evitar violaciones a sus derechos.

Las mujeres, jóvenes y niñas están dentro de los grupos expuestos a mayores violaciones y, por lo general, son víctimas de explotación laboral en actividades del trabajo doméstico y servicios sociales.

A diferencia de los años ochenta y noventa-y durante la crisis económica y financiera-hoy los procesos migratorios no solamente se dan hacia los países desarrollados, sino también a lo interno de los propios territorios y hacia países en vías en desarrollo que muestran indicadores de crecimiento u oportunidades de empleo.

Hay que proteger los derechos humanos de los migrantes a través de las normas del derecho internacional y reconocer sus aportes en los países de origen y en los receptores.

Se estima que la cantidad de migrantes internacionales alcanza en todo el mundo, los 175 millones, los que equivalían aproximadamente al 3\% de la población mundial en el año 2000. En el caso de América Latina y el Caribe, hacia, desde y a través del continente americano, se dificulta la recolección de información, el número de migrantes en América Latina y el Caribe creció en 5 años en 4 millones de personas llegando a 25 millones de inmigrantes en el 2005. Aunque esta cifra solamente representa el 1.1\% de la población regional, el impacto sobre el Caribe ha sido más fuerte, considerando la heterogeneidad en el patrón migratorio. (CEPAL/CELADE, 2006)

Desde la Declaración Universal de los derechos humanos, se garantizan el derecho de las personas a la libertad de circulación y elección de su residencia en cualquier lugar, y a salir de su país de origen. Así las cosas, los y las migrantes están amparadas en las normas internacionales que obligan a los Estados a respetar sus derechos, incluyendo a los refugiados o desplazados, aun cuando el tratamiento jurídico sea distinto.

Pero lo preocupante es que, a pesar de la existencia de acuerdos migratorios, las violaciones hacia las personas migrantes es una realidad que crece y se agrava para los grupos más vulnerables, especialmente las mujeres cuya decisión de emigrar obedece a distintos factores, aunada a la migración forzada, cuyas consecuencias ponen en riesgo la vida de las personas, pero que amerita que sea considerada como parte importante de la agenda de derechos humanos, y no transversalmente.

\section{Igualdad entre los géneros y eliminación de todas las formas de discriminación.}

La desigualdad tiene múltiples expresiones: del ingreso, étnica, territorial y la de género, que impide la

posibilidad a millones de mujeres ejercer sus derechos y alcanzar un empoderamiento físico, político y económico: se trata de que alcancen su autonomía y decidan sobre sus propias vidas. 
Las relaciones de poder entre hombres y mujeres, la subordinación histórica y las violaciones a los derechos de las mujeres, desde el análisis de género como un derecho humano autónomo; nos facilita comprender las necesidades diferenciadas y específicas de las mujeres y hombres, así como las discriminaciones que se tejen alrededor de la construcción androcéntrica del mundo, que lesiona la vida, la dignidad y las oportunidades de las mujeres, de todas las edades, y clases sociales.

La discriminación contra la mujer y la subordinación tienen repercusiones trascendentales en la vida cotidiana de hombres y mujeres, sin importar etnia, condición social, educación, nacionalidad o creencia u orientación sexual. Las mismas persisten y se reproducen por medio de una serie de estructuras sociales y mecanismos culturales_ como el lenguaje, la música, la historia, la literatura—que denigran la imagen de la mujer y subordinan su papel a lo doméstico con limitación a sus derechos para participar en la toma de decisiones familiares, políticas y comunitarias.

A partir del reconocimiento que cuando hablamos de género se hace referencia a los valores, atributos, roles y representaciones que la sociedad le asigna a hombres y mujeres, podemos identificar las asimetrías de género y cómo las ideas de superioridad masculina tienen impactos negativos en las relaciones interpersonales y sociales, en la división sexual del trabajo, el acceso y control de los recursos y servicios y la profundización de las desigualdades de género.

Compartimos lo expresado por Lagarde al señalar que:

La perspectiva de género implica una mirada ética del desarrollo y la democracia, como contenidos de vida para enfrentar la inequidad, la desigualdad, los oprobios de género prevalecientes. Es decir, la perspectiva de género es una toma de posición política frente a la opresión de género: es una denuncia de sus daños y su destrucción y es, a la vez, un conjunto de acciones y alternativas para erradicarlas. (Lagarde, 1996, Pág.20)

Una agenda de derechos humanos, bajo la persistente desigualdad en América Latina y el Caribe, debe procurar una igualdad real entre los géneros, donde ambos sean actores protagónicos del desarrollo y tengan por igual el acceso a los recursos y beneficios con equidad; pero que sobre todo, apunte a transformar las relaciones y la cultura que la reproduce, encubre y sustenta, porque esta discriminación de género, está presente en todas los ámbitos de los derechos humanos.

Según la CEPAL "la desigualdad y por tanto la falta de autonomía de las mujeres, son principalmente, consecuencia de la injusticia, de la mala distribución del poder, los ingresos y el tiempo entre hombres y mujeres, y de la falta de reconocimiento de los derechos de las mujeres". (Dirk Jasper-Faijer, 2014)

Se habla mucho del ODM3, referente a la igualdad y equidad entre los géneros, cuyas metas debían alcanzarse, sobre todo en materia de educación para las niñas y paridad política. La realidad nos confronta con la persistente desigualdad que nos dice que, aun cuando se reconoce como un tema transversal, si queremos eliminarla debe ser atendida de manera autónoma en toda la agenda. 
Invest. pens. crit.

Vol. 3, No. 3, septiembre-diciembre 2015

pp. 38-61

En América Latina se han dado algunos pasos favorables para ir cerrando las brechas de desigualdad de género. Lo interesante es que en los países donde se muestran mayores logros, no necesariamente registran los más altos indicadores de desarrollo humano, (IDH), ni el mayor crecimiento económico; lo que nos daría la razón al plantear que eliminar las causas culturales y estructurales de la desigualdad entre los géneros requiere que las políticas públicas que adopten los Estados, tengan un enfoque de respeto a los derechos humanos, establezcan como prioridad su erradicación, y tengan la voluntad política de mantenerla como tema de Estado, que trascienda planes de gobierno.

Las políticas públicas en términos generales, siguen ciegas al género, y a un enfoque de derechos humanos, ya que no analizan el papel de las mujeres como generadoras de ingresos, la relación existente entre trabajo productivo y reproductivo, y la contribución de la economía doméstica a la riqueza. Las políticas públicas siguen presentando falencias para promover una equidad de género, que por un lado genere cambios estructurales en las formas en que hombres y mujeres se relacionan en los actuales modelos económicos. (Fries, 2008, Pág.13)

En el reporte de Brecha Global de Género del Foro Económico Mundial se reconoce que al mundo le tomaría por lo menos 81 años cerrar las brechas de género y, a pesar de la creciente participación de las mujeres en el mundo laboral y la economía, en ningún país la mujer cobra tanto como el hombre.

Entre los países que muestran avances en igualdad y equidad de género de América Latina, entre 20 seleccionados en el mundo, se encuentran Nicaragua en el lugar No.10 y Cuba en el No.15-que pasarían a ser los primeros de nuestra región—seguidos de Ecuador en el 25, Bolivia 27, Barbados 29, Costa Rica 31, Argentina 34, Colombia 35, Trinidad y Tobago 36 y Panamá en el No.37, Brasil en el No.62 y México en el No. 68.

El informe mide el tamaño de la brecha de desigualdad de género en cuatro áreas:

1. Participación económica y oportunidad: salarios, participación y empleo altamente capacitado

2. Logros educativos : acceso a niveles de educación básicos y más elevados

3. Participación política: representación en las estructuras de toma de decisiones

4. Salud y supervivencia: expectativa de vida y proporción hombres-mujeres. (Cann, 2013).

La desigualdad de género se expresa, además, en la participación política de las mujeres em cargos de elección popular, cargos públicos y directivos a nivel privado. La región experimentó avances con las leyes de cuotas en casi todos los países, pero la paridad política sigue siendo una deuda pendiente con la mitad de la población. Ser parte en la toma de decisiones, compartir el poder desde la mirada feminista, aportará mucho al desarrollo humano sostenible. La presencia de mujeres en altas magistraturas como ha sido las experiencias de Presidentas en Panamá, Nicaragua, Costa Rica, Brasil, Argentina, Chile, no significa que se han dado las condiciones para producir esos cambios culturales y estructurales. 
Cerrar esta brecha y otras relacionadas con la etnia, edad, condición de la mujer, nos demanda darle un tratamiento de discriminación positiva a los derechos humanos de las mujeres, para que puede alcanzar las autonomías a que tiene derecho. Y vivir sin el temor a ser víctima de la violencia.

\section{Derechos de los grupos más vulnerables, poblaciones indígenas y afrodescendientes.}

La humanidad ha reprobado la materia de garantizar los derechos humanos, civiles, políticos, sociales, culturales, ambientales y colectivos; pero tenemos una nueva oportunidad de replantear los objetivos del desarrollo más allá del 2015. Es un llamado a articular un discurso coherente con la realidad, superar los enfoques tradicionales economicistas, evaluar la proliferación de objetivos, establecer alianzas inclusiva y mecanismos de rendición de cuentas, trascender las prioridades de los países desarrollados, para privilegiar los problemas del desarrollo humanos sostenible, la desigualdad que afecta principalmente a los grupos vulnerables, poblaciones indígenas y afrodescendientes de los países en vía de desarrollo.

Es hora de decidir cómo enfrentar los complejos problemas del ser humano y de la sociedad, desde la responsabilidad de todos, con solidaridad, respeto a la multiculturalidad, ancladas en los pilares de los derechos humanos, desde una visión holística.

La resistencia de los pueblos originarios no ha sido suficiente para que se reconozcan sus demandas. El despojo de territorio, la exclusión social, la pobreza, la desigualdad y la persistente violación de sus derechos a los que están sometidos han sido parte cotidiana de la vida de millones de indígenas. Década tras década promesas incumplidas y el progreso y crecimiento económico sostenible para la región, no los alcanza.

Los pueblos indígenas siguen al margen de los beneficios del desarrollo, aun cuando en casi en todos los Estados se obligan a adoptar políticas públicas_a la luz de la Declaración aprobada por la Asamblea General de las Naciones Unidas_sobre los derechos de los pueblos indígenas en el 2007 y de conformidad con los acuerdos gubernamentales plasmados en el Consenso de Montevideo, aprobado en la Primera Conferencia de Población y Desarrollo, de América Latina y el Caribe en el 2013.

En las luchas por el reconocimiento de sus derechos ha sido importante la influencia del Sistema Interamericano de Derechos Humanos, especialmente, en los pronunciamientos de la Comisión Interamericana de Derechos Humanos y la Corte Interamericana, sobre los derechos de las poblaciones indígenas, a su territorio, tierras y recursos naturales. Las reclamaciones tienen como objeto de disputas, que los Estados anteponen los derechos económicos y comerciales y otorgan concesiones para la explotación de las actividades mineras, hidrocarburos y utilización de los recursos naturales, en contraposición a sus derechos colectivos.

El Convenio 169 de la Organización Internacional del Trabajo (OIT), Sobre Pueblos Indígenas y Tribales en países Independientes, reconoce derechos a los pueblos indígenas entendiendo su diferencia 
Invest. pens. crit.

Vol. 3, No. 3, septiembre-diciembre 2015

pp. 38-61

cultural y autoindentificación, su derecho al territorio, a tener su propia autoridad y justicia en sus territorios.

A nivel constitucional, existen avances en la mayoría de los países latinoamericanos y caribeños, donde se consagra el derecho de los pueblos indígenas a su patrimonio cultural, idioma y autoridades tradicionales.

La autonomía o libre determinación que reclaman los pueblos indígenas, implica el reconocimiento de sus territorios, idioma, cultura, recursos, justicia, organización social y política, medicina tradicional o conocimientos ancestrales.

Uno de los ejemplos de estas luchas por la autonomía de los pueblos indígenas, fue en Panamá con la Revolución Tule, en el año 1925, donde la Comarca Guna Yala, logro el reconocimiento del Estado, de su cultura, tradiciones, idioma, territorio y autoridades tradicionales.

Las comunidades indígenas tienen una relación especial con la naturaleza y son protectores de nuestros recursos y han acumulado conocimientos importantes ancestrales del que debemos aprender y respetar.

Existe una deuda histórica con las poblaciones indígenas: su marginación social y la violación de sus derechos, son intolerables y una vergüenza en Estados que se consideran democráticos. En América Latina la situación grave de vulnerabilidad de los derechos humanos se encuentra en estas poblaciones, que siguen siendo discriminados social y económicamente, con ingresos exiguos, pobreza extrema, peores condiciones de salud, educación, violación de sus territoriales, patrimonio cultural y menosprecio a las demandas de sus autoridades tradicionales.

Según información censal disponible de la CEPAL, se puede decir que en la actualidad en América Latina existen más de 826 pueblos indígenas, que representan alrededor de 46 millones de personas. Entre los países con mayor representación se encuentran Brasil con 135 pueblos indígenas, Colombia con 102, Perú con 85, México con 78, Bolivia 39 y Costa Rica con 9 y Panamá con 7 (Jaspers-Faijer, Del Popolo y Reboiras, 2014).

Con independencia de la cantidad de población, los Estados latinoamericanos no pueden continuar postergando la atención a las poblaciones indígenas y afrodescendientes. La agenda para el desarrollo es una oportunidad, para generar políticas públicas, desde un enfoque holístico y de derechos humanos; de lo contrario, sería ignorar los más de 120 millones de personas. Es un imperativo ético y de justicia social, que sean la prioridad en esta nueva coyuntura, donde los Estados se reúnen para decidir el futuro del desarrollo de nuestros pueblos. Sería inaceptable otra década de menosprecio y desamparo de las poblaciones latinoamericanas por razones de etnia, género, territorio o condición social.

Han sido históricas las luchas de los pueblos originarios por sus reivindicaciones, que se sintetizan en cinco aspectos prioritarios mínimos que deben cumplir los Estados; la no discriminación; desarrollo y 
bienestar social; integridad cultural; propiedad, uso, acceso y control de la tierra, territorios y recursos naturales y la participación política.

La situación de las personas afrodescendientes(descendientes de africanos) en las América-estimada en un 30\% del total de la población regional, que representa a más de 75 millones-nos acerca a la cruda realidad que viven nuestros hermanos, en distintas regiones, urbanas y rurales, quienes comparten con las poblaciones indígenas, la discriminación racial, la desigualdad social y económica y pobreza. Si bien han logrado que algunas de sus demandas sean incorporadas en las políticas públicas, aún falta mucho para que se reconozca el carácter multiétnico y pluricultural de nuestras sociedades. (OEACIDH, 2011)

Las desigualdades étnico raciales están presente en cada una de las metas que se pretenden alcanzar. En este sentido, cualesquiera que sean los objetivos convenidos entre los Estados, deben tener una lectura integral, considerando la pluriculturalidad y etnicidad, presente en nuestras sociedades.

\section{Protección y prevención de los efectos del cambio climático y seguridad alimentaria.}

Uno de los problemas más complejos que enfrenta la humanidad es el cambio climático. Los Estados deben dedicar esfuerzos para adoptar diversas acciones integrales y sostenibles como una prioridad en la nueva agenda de derechos humanos. El debate, análisis y decisiones deben estar enfocados a prevenir los riesgos a la vida, integridad y bienes de las personas, expuestas a los desastres. Los efectos de este problema no tiene nacionalidad, ni fronteras: las consecuencias son de impacto global y pueden llevar a la destrucción de nuestro planeta.

La alianza entre países con intereses geopolíticos y económicos diversos, desarrollados o en vías de desarrollo, tanto de Europa como de América Latina, ha permitido generar espacios de debate multilateral para la protección del clima. Sin embargo, los resultados de Kyoto, Copenhague en 2009, Durban y Río en 2012, no son alentadores ya que aún no se concretan acuerdos para adoptar un sistema de normas internacionales amplio, vinculante y equitativo.

No se puede seguir siendo un espectador ante los depredadores del ambiente y los recursos. Cada año se pierden millones de áreas boscosas, las fuentes de aguas son más escasas, las especies están en peligro de extinción, los contaminantes agotan la capa de ozono y las emisiones de dióxido de carbono aumentan de forma alarmante.

Sin la voluntad política de los países desarrollados es imposible lograr una economía baja en carbono y una inversión capaz de mitigar los daños producidos y prevenir mayores desastres que, a la postre, tendrá efectos inimaginables para todo el mundo. La naturaleza no distingue: la capacidad de dialogar debe mantenerse y la participación ciudadana y de las organizaciones de la sociedad civil, deben contribuir en los esfuerzos de concienciar sobre la gravedad del problema.

América Latina—y particularmente Centroamérica—es más vulnerable frente al cambio climático: 
Invest. pens. crit.

Vol. 3, No. 3, septiembre-diciembre 2015

pp. 38-61

Diferentes grupos reclaman la necesidad de priorizar zonas de ecosistemas que proveen sustento para sus comunidades, haciendo referencia a la adaptación de los ecosistemas que es necesaria para una amplia gama de beneficios, tales como la provisión de recursos de pequeñas comunidades la protección de agua, y la protección ante desastres. (Verolme, Vasconi Reca y Maurtua Konstantinidis, 2014)

El establecimiento de asentamientos de población en áreas susceptibles a desastres naturales o a mayores impacto ante fenómenos climáticos, aunado a la falta de planificación, los hábitos productivos, la falta de acceso a los servicios e infraestructuras, políticas de vivienda y sanidad básica, colocan en situación de permanente riesgos a estas personas.

La drástica reducción de las emisiones de carbono para prevenir el cambio climático y el aumento del nivel de los mares puede suponer que incluso los niveles actuales de consumo sean insostenibles, en particular si la calidad de vida de las regiones más pobres y de las economías emergentes aumenta. (Wikilson \& Picket, 2009)

No hay forma de establecer el impacto económico de los desastres en la vida de las personas: las estimaciones, no recogen la gravedad psicológica y social que estos eventos naturales causan, especialmente, a las personas más vulnerables.

La pérdida en vidas, en infraestructuras, es lo que más impacta, pero ¿qué sucede con los que no tienen alternativas para recuperarse de los desastres? No solo es perder las pocas cosas que tienen, sus hogares, áreas donde vivir, sus animales y cultivos de las cuales dependen para su subsistencia.

Generalmente, los pobres se mantienen en las mismas áreas con la angustia de saber que sus vidas están en riesgo, pero en la encrucijada de no tener recursos ni respuestas integrales que les permita iniciar su vida con mayores oportunidades: no tienen otra salida. Las ayudas o no llegan o cuando se entregan son escasas y al poco tiempo el Estado se olvida de las necesidades de estas poblaciones.

La seguridad alimentaria es otro de los temas esenciales en la nueva agenda de derechos humanos en América Latina y el Caribe. Los costos en materia de vida, salud, bienestar, limitación a las capacidades de aprendizaje y producción económica, resultan inestimables.

A pesar que en la Cumbre Mundial sobre la Alimentación de 1996 se reafirmó el derecho de toda persona a tener acceso a alimentos sanos y nutritivos, y los Estados allí reunidos decidieron que se debía aclarar el contenido de los derechos relacionados con la alimentación contemplados en el Pacto Internacional de Derechos Económicos, Sociales y Culturales, estos compromisos y su seguimiento aún no tienen este enfoque en las políticas públicas, lo que no puede quedar al margen de los debates en la nueva agenda, para lograr su incorporación.

El ambiente y la alimentación, aunque son derechos autónomos, se vinculan permanentemente. Las amenazas ambientales están presentes en todos los países, no solo los vinculados a los fenómenos del niño o la niña, que dejan a su paso inundaciones, sequías, e incontables pérdidas para la agricultura, la 
ganadería, las economías de las personas y los países. Otras afectaciones de la naturaleza que inciden en la producción de alimentos son: la degradación de los recursos naturales de tierras, aguas y cuencas hidrográficas, y el impacto de los desastres en las áreas rurales empobrecidas, dedicadas a una agricultura de subsistencia, granjas, o de pequeños productores.

Como resultado de la Cumbre Mundial sobre Alimentación, se adoptaron acuerdos importantes para la acción:

La Declaración de Roma sobre la Seguridad Alimentaria mundial y el Plan de Acción de la Cumbre Mundial sobre la Alimentación establecen las bases de diversas trayectorias hacia un objetivo común: la seguridad alimentaria a nivel individual, familiar, nacional, regional y mundial. Existe seguridad alimentaria cuando todas las personas tienen en todo momento acceso físico y económico a suficientes alimentos inocuos y nutritivos para satisfacer sus necesidades alimenticias y sus preferencias en cuanto a los alimentos a fin de llevar una vida activa y sana. A este respecto, es necesaria una acción concertada a todos los niveles. Cada país deberá adoptar una estrategia en consonancia con sus recursos y capacidades para alcanzar sus objetivos propios y, al mismo tiempo, cooperar en el plano regional e internacional para dar soluciones colectivas a los problemas mundiales de la seguridad alimentaria. (Diouf, 1996.)

El derecho a la alimentación garantiza nuestra sobrevivencia; la hambruna que se cierne sobre la humanidad, no son voces agoreras. La Organización de las Naciones unidas para la Agricultura y la Alimentación (FAO), en su informe temático de la agenda al desarrollo Post- 2015 divulgado en medios de comunicación social, señaló que al menos el $12 \%$ de la población mundial—unos 842 millones de personas - se van a dormir con hambre. Esto implica actuar en todas las dimensiones. La seguridad alimentaria debe entenderse en cuatro dimensiones como son la disponibilidad, acceso, la estabilidad y la utilización.

Una nutrición adecuada desde la primera infancia desarrolla las capacidades de aprendizaje y es una necesidad vital: es parte del derecho de alimentación de la población, que está empalmada al derecho a la salud. En otras palabras, no es suficiente que se garantice la atención médica o los medicamentosservicios a los que indiscutiblemente tengo derechos-si no tengo cómo obtener los alimentos.

Desde el enfoque de derechos humanos, se requiere que el Estado tenga políticas públicas para que los grupos vulnerables tengan recursos y acceso a los alimentos, realice las inversiones para la soberanía alimentaria, que no requiera de los mercados externos para suplir a la población de alimentos básicos; e implemente planes para enfrentar los riesgos de desastres.

En un mundo con tantos recursos económicos, es inaceptable que millones de personas mueran de hambre y no tengan acceso al agua, que se convierte en un derecho humano emergente. Igualmente inaceptable es que no se adopten políticas públicas que aseguren la alimentación. Hay que reconocer la necesidad de revisar profundamente, las decisiones que los diversos Estados están adoptando en materia de desarrollo económico y social. 
Invest. pens. crit.

Vol. 3, No. 3, septiembre-diciembre 2015

pp. 38-61

$\mathrm{Al}$ incluir en el debate estos derechos humanos de forma autónoma, nos permitirá avanzar, pero sin dejar de entrelazarlos con los demás derechos; es lo que llamamos la "escalera de derechos (Figura $1)^{\prime \prime}$.

Dicho esto, consideramos que debemos exigir la protección y promoción, no como un derecho de otra generación, sino integrados e inseparables, porque todos son determinantes, comparten su universalidad, e indivisibilidad, están unidos en eslabones inseparables. La vida, integridad, libertad, igualdad, acceso a la justicia, los derechos económicos, políticos y sociales y colectivos, equidad de género, hacen un todo. Al final de la escalera, todas las metas se alcanzan, un ser humano en goce y ejercicio pleno de todos sus derechos.

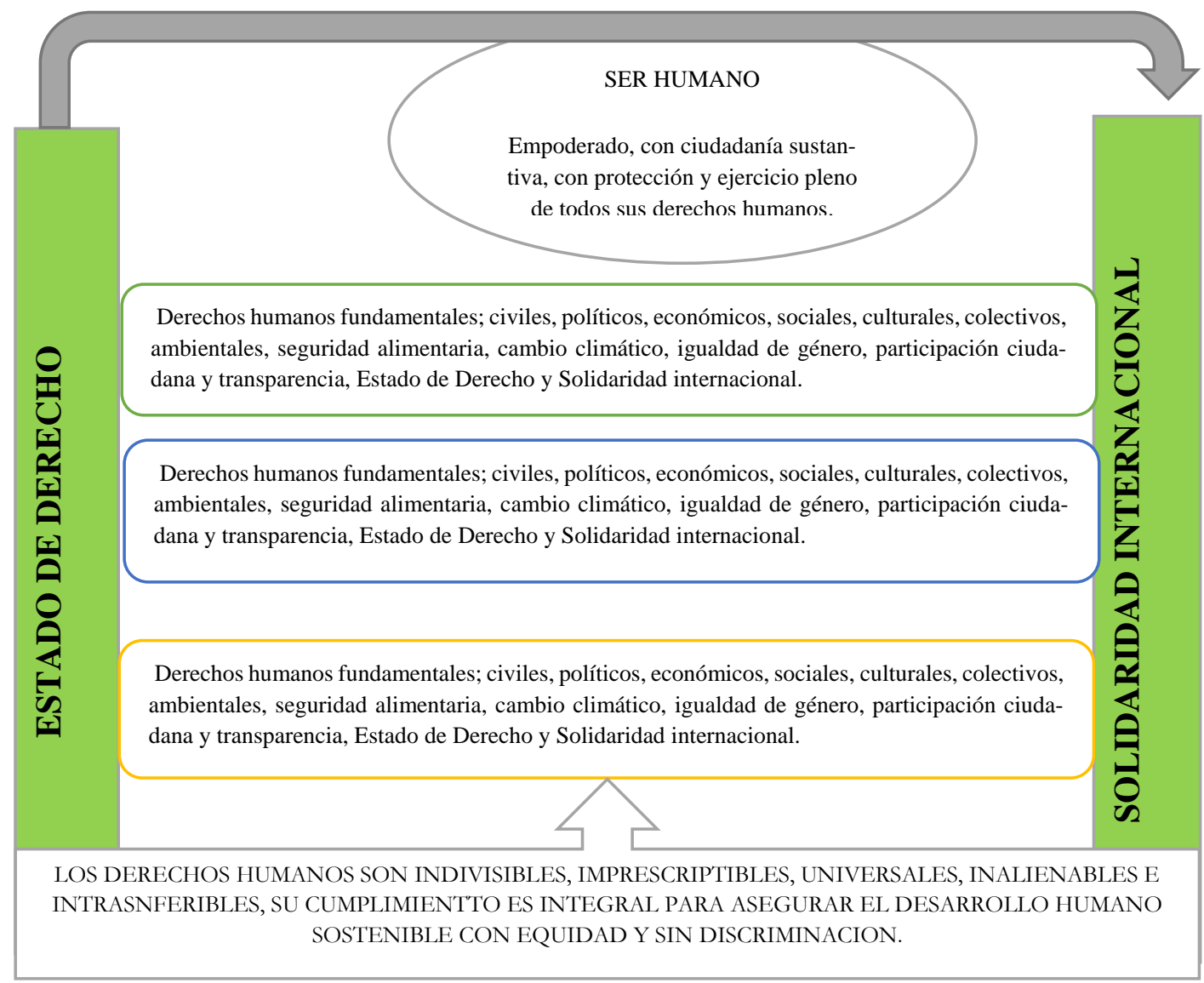

Figura 1. Escalera de derechos humanos. Elaboración propia.

Cada uno de los derechos humanos pueden compararse al ser humano, formado por distintas partes del cuerpo, donde cada uno es tan importante como el otro, pero hacen un todo indivisible. Para vivir plenamente en su dignidad, requieren de atención integral, como ser individual y social. Esto no se contrapone con la necesidad de que cada uno de los instrumentos internacionales de derechos humanos tenga sus propios mecanismos especializados de monitoreo y rendición de cuentas. 


\section{Participación ciudadana y transparencia.}

La participación ciudadana es un derecho humano de carácter político donde, a través de distintos mecanismos, las personas, las organizaciones o expresiones organizadas de la comunidad, se vinculan a la administración pública o municipal y forman parte en la toma de decisiones para que el Estado ejerza sus competencias en el marco de legalidad y transparencia, atendiendo su principal responsabilidad de trabajar por el bien común de la población y la protección de sus derechos.

Existen avances en el reconocimiento del derecho a la participación ciudadana. La sociedad civil, los movimientos sociales, las organizaciones de mujeres y la comunidad, han propiciado cambios importantes exigiendo estos derechos y mejorando la interlocución con el Estado. De igual forma, las normas internacionales son herramientas valiosas en este aspecto, aunque hay una resistencia de aperturas de los asuntos públicos y en las decisiones que le afectan sus derechos.

En materia constitucional y legal se le reconoce el derecho a las personas para organizarse, asociarse o individualmente ejercer una participación sustantiva en distintos aspectos de la vida política, social, cultural, económica y ambiental, a nivel nacional y local. Lo que se busca con el respeto al derecho a la participación ciudadana es que el Estado lo considere como aliado para el buen gobierno, en la toma de decisiones, en su ejecución, control, beneficios y construyendo con sus ideas a una mejor sociedad.

Aunque de manera formal existen estas instancias de participación, en la práctica son espacios cuestionados porque, una vez instalados, en algunos casos los representantes de la sociedad no hacen rendición de cuentas. Existen diversas expresiones de participación ciudadana como consejos nacionales y locales para atender diferentes problemas de los grupos vulnerables; el ejercicio del sufragio, asociaciones, peticiones, reuniones, cabildos, comisiones, juntas consultivas, asambleas, plebiscitos, planificación de programas y proyectos públicos, elaboración de presupuestos y las iniciativas ciudadanas de propuestas de ley.

El líder y ex presidente Africano, Nelson Mandela, en su autobiografía, "El largo Camino hacia la Libertad," comparte sobre la expresión pura de la democracia que se practicaba en los procedimientos de las reuniones locales, donde todo el que quería hablaba y era escuchado, sin importar su jerarquía. De esto se trata la participación ciudadana, de ejercer tu derecho a ser escuchado y ser parte en la toma de decisiones; de gobernar con legitimidad, pensando en el bienestar de la población, quien tiene los espacios para plantear sus intereses y necesidades.

En la actualidad, los sistemas políticos se encuentran retrasados respecto del acelerado dinamismo de los cambios que experimenta la sociedad. Uno de los indicadores más sobresalientes en este sentido es que, a pesar de que la sociedad latinoamericana como han mostrado variadas encuestas valora y defiende la democracia, se siente crecientemente defraudada por la ineficacia e ineficiencia del sistema político. Duda cada vez más de la legitimidad de éste para representar sus intereses, a la vez que demanda mayores niveles de eficiencia y efectividad del Estado y la generación de espacios públicos para la participación, el dialogo y la deliberación. (Fernando, 2004). 
Invest. pens. crit.

Vol. 3, No. 3, septiembre-diciembre 2015

pp. 38-61

La Declaración Universal de Derechos Humanos de 1948, la Convención Americana de Derechos Humanos, Pacto de San José de Costa Rica, El Pacto Internacional de Derechos Civiles y Políticos reconocen el derecho a la participación ciudadana, en los asuntos públicos, en el acceso a cargos, a representar al Estado, a formar parte de las decisiones que afectan su vida.

La Carta Democrática Interamericana constituye uno de los principales instrumentos del Sistema Interamericano y, en su artículo 6, establece lo siguiente: "La participación de la ciudadanía en las decisiones relativas a su propio desarrollo es un derecho y una responsabilidad. Es también una condición necesaria para el pleno y efectivo ejercicio de la democracia. Promover y fomentar diversas formas de participación fortalece la democracia."

La democracia requiere de la participación ciudadana. Aunque se vea de distintas clases en sí misma, es un concepto en construcción y evolución. Si hablamos de la democracia de los ciudadanos o participativa, los ciudadanos son actores protagónicos y su perfeccionamiento conlleva que los gobernantes entiendan que el ejercicio del poder, está sometido a la soberanía que radica en el pueblo.

Las aperturas a la participación de las organizaciones de la sociedad civil vinculadas a la defensa de los derechos humanos, en los foros internacionales y nacionales y sus aportes y experiencias, son alentadores, pero no suficientes. El mayor aliado de los Estados y de la democracia, son precisamente los ciudadanos, el ser parte de la toma de decisiones, le brinda herramientas para concertar temas que afectan a todos; permiten más transparencia en la instituciones, autoridades y que la democracia funcione en beneficio de las grandes mayorías.

Esta participación ciudadana efectiva propicia los diálogos y consensos sobre temas relevantes para la sociedad, facilita la veeduría social, la transparencia y la rendición de cuentas y, quizás lo más importante, devuelve la confianza de los ciudadanos en las instituciones y al sistema democrático, porque en el ejercicio del poder cometen abusos, no siempre toman las mejores decisiones y cumplen las expectativas de respeto a nuestros derechos personales, colectivos. Como señala Rojas Aravena:

El desencanto que se observa en la ciudadanía, con todo lo que comúnmente se relaciona con la política, genera el riesgo de que se pierda el sentido de entender la función y el para qué de los políticos o peor aún, para qué algunas instituciones fundamentales de la democracia.

Los avances en las democracias latinoamericanas no se consolidaran, ni serán permanentes, sino se refuerza, la cultura política y las formas y prácticas de la democracia misma. (Rojas Aravena, 2013, Pág.38)

Una nueva agenda de los derechos humanos debe apostar a formar lo que llamaríamos un ciudadano/a virtuoso/a, con capacidad de ser crítico y reflexivo, creativo y propositivo, que exige sus derechos y respeta los de sus semejantes, cumple sus responsabilidades ciudadanas, orgulloso de su identidad, con consciencia ambiental, ética y solidaridad humana. 
Desde el enfoque de derechos humanos, debemos potenciar las capacidades de las personas y brindarle oportunidades en un Estado fortalecido, capaz de generar estrategias integrales, sin discriminación y en pleno respeto a los derechos humanos, consciente de su responsabilidad de transparentar sus actuaciones.

Cuando las sociedades tienen ciudadanos virtuosos/as, los gobiernos y sus instituciones son más prolijas a ser transparente y se logran instrumentos para evitar conductas que lesionen los derechos humanos.

Cada vez es más importante el respeto al derecho humano a la información en manos del Estado, sus instituciones u organismos sobre el manejo de la gestión pública. También es necesario se tenga acceso a las reglas, normas, requisitos que adoptan las instituciones para dar respuestas a las peticiones de las personas naturales o jurídicas, así como el uso que se le dan a los recursos públicos, o en temas relacionados a la justicia o personas privadas de libertad.

\section{Referencias}

Cann, O. (25 de octubre de 2013). World Economic Forum. América Latina cierra brechas de género. Disponible en http://www.webforum.org

CEPAL/CELADE. (2006). Migración internacional derechos humano y desarrollo en América Latina y el Caribe. Sintesis y conclusiones. Montevideo, Uruguay: CEPAL.

Chen, S. (10 de agosto de 2012.Pág.2). Comité Asesor del Consejo de Derechos Humanos. ONU. Disponible en http://www.ohchr.org/doc/sesion 9

Desarrollo.PNUD, P. d. (24 de junio de 2014). ¿Cómo esta América Latina y el Caribe? Disponible en http://wwwrevistahumanum.org

Diouf, J. (13 de noviembre de 1996.). Cumbre Mundial de la Alimentación. Plan de Acción de la Cumbre de la Alimentación. Disponible en http://www.fao.org

Dirk Jasper-Faijer, S. P. (2014). Propuesta de Agenda regional en población y desarrollo para América latina y el Caribe 2014. Santiago, Chile: CEPAL, CELADE.

EFE. (18 de diciembre de 2014). Texto del Discurso de Obama, sobre la reanudación de las relaciones con Cuba. El Confidencial, págs. 1-2.

Essayag, S. (2013). El Compromiso de los Estados: Planes y Políticas para erradicar la violencia contra las mujeres en América Latina y El Caribe. Panamá: ONU Mujeres-PNUD. 
Invest. pens. crit.

Vol. 3, No. 3, septiembre-diciembre 2015

pp. 38-61

Felipe, G. (2004). Crisis de la política: causas y respuestas eficientes. En P. d. Desarrollo, La Democracia en América Latina. Hacia una democracia de ciudadanas y ciudadanos (págs. 41-72). Buenos Aires, Argentina: Aguilar, Altea, Taurus, Alfaguara.

Fernando, C. (2004). Notas sobre la crisis de legitimidad del Estado y la democracia. En P. d. Desarrollo, La Democracia en América Latina. Hacia una democracia de ciudadanas y ciudadanos (págs. 193-213). Buenos Aires, Argentina: Aguikar, Altea, Alfaguara.

Fitoussi, J.-P. y Rosanvallon, P. (2003). La nueva era de las Desigualdades. Buenos Aires, Argentina: Manantial.

Foucault, M. (1980). Microfísica del poder. Madrid, España: La Piqueta.

Fries, L. (2008.Pág.13). Informe Regional de Derechos Humanos y justicia y Género. Ecuador: Andros impresores.

García Roco Pérez, O. (2002). La Salud Pública en América Latina en América Latina y el Caribe. Humanidades Medicas, 2(2), 3-5.

Jara, L. y Roberts, E. G. (2009). La salud de las mujeres y los hombres en las Américas. Washington, EEUU: OPS/OMS.

Jaspers-Faijer, D., Del Popolo, F. y Reboiras, L. (2014). Los pueblos Indígenas en América Latina. Avances en el último decenio y retos pendientes para garantizarle sus derechos. Santiago, Chile: CEPAL-CELADE.

Lagarde, M. (1996. Pág.20). Género y feminismo. Desarrollo bumano y democracia. Madrid, España: Horas y HORAS. .

Larre, M. L. (2005). "Reformas neoliberales en América Latina”: Argentina y Venezuela. Las reformas de los presidentes Carlos S. Menem y Carlos. A. Pérez en perspectiva comparada. Revista de Ciencia Política No.3., 1-7.

Miller, T., Saad, P., Martínez, C. y Calvo, J. J. (14 de Noviembre de 2014). La Nueva Era Democrática de América Latina y el Caribe. Documento de referencia de la primera Reunión de la Mesa Directiva de la Conferencia Regional de Población y Desarrollo. Santiago, Chile, Latinoamérica y el Caribe: CEPAL/CELADE.

Moon, B.-K. (2014). Objetivo de Desarrollo del Milenio. Informe 2014. New York: ONU-Secretaria General.

Mundo, E. (18 de Diciembre de 2014). Discurso integro de Raúl Castro tras el restablecimiento de las relaciones con Cuba. El Mundo, págs. 1-2.

Nikken, P. (1994.Pág.17). El concepto de Derechos Humanos. Tomo I. San José, Costa Rica: IDDH. 
Rojas Aravena, F. (2013.Pág.38). Nuevo Contexto Global y Mapa Político Latinoamericano. En A. Bonilla y M. Ortis Salvadora, El Papel Político, Económico, Social y Cultural de la Comunidad Iberoamericana, en un Nuevo Contexto mundial: aportes de un debate en curso (págs. 27-44). San José, Costa Rica: SEGIB-PNUD.

UNESCO. (2015.Pág.5). Agenda Educacional Post-2015. América Latina y el Caribe. Santiago, Chile: UNESCO.

UNFPA. (mayo de 2008). Fondo de Población de las Naciones Unidas. Adolescencia y Juventud. Disponible en http://www.unfpa.org

Unidos por los Derechos Humanos. (10 de diciembre de 2008). United For Human Rights. Disponible en: $\quad$ http://www.humanrights.com/es ES/what-are-human-rights/brief-history/cyruscylinder.html

Verolme, J. H., Vasconi Reca, P. y Maurtua Konstantinidis, E. (2014). Europa y América Latina de cara a una acción colectiva más ambiciosa sobre el clima. Bélgica: Heinrich Boll Stiftung.

Wikilson, R. y Picket, K. (2009). Desigualdad. Un análisis de la (in)fedilidad colectiva. Madrid, España: Turner Publicaciones.

Williamson, J. (2003). No hay consenso. Reseña sobre el Consenso de Washington y sugerencias sobre los pasos a dar. Finanzas y Desarrollo, Fondo Monetario Internacional, 1-4. 